\title{
Preliminary design and multi-objective optimization of electro-hydrostatic actuator
}

Proc IMechE Part G

J Aerospace Engineering

000(00):1-13

(CThe Author(s) 2010

Reprints and permission:

sagepub.co.uk/journalsPermissions.nav

DOI:doi number

http://mms.sagepub.com

\section{Shuai Wu, Bo Yu, Zongxia Jiao, Yaoxing Shang \\ School of Automation Science and Electric Engineering, Beihang University, Beijing, 100191 China \\ National Key Laboratory of Science and Technology on Holistic Control, Beihang University, Beijing, 100191 China}

\section{P. C. K. Luk}

Electric Power and Drives Group, Power Engineering Centre, Cranfield University, Bedfordshire, UK

\begin{abstract}
Electro-hydrostatic Actuator (EHA) is a key power-by-wire (PBW) technology for more electrical aircraft (MEA). It is important that interesting to optimize it performances, such as weight and efficiency, in the preliminary design phase. This paper describes a multi-objectives optimization (MOO) preliminary design method of EHA with the objectives of weight and efficiency. Models are developed to predict weight and efficiency of EHA from the requirements of control surface. The models of weight prediction are achieved by using scaling laws with collection data, and the efficiency is calculated by the static energy loss model. The multi-objectives optimization approach is used to find the Pareto-front of objectives and the design parameters. A case is studied to optimize a EHA with the given requirement of control surface. The optimized results indicate the selection space and the performance trend of key design parameters for preliminary design. The proposed approach can explore the influence of level length, pump displacement and motor torque constant on the weight and efficiency of the actuator.
\end{abstract}

Keywords

More Electric Aircraft, Electro-hydrostatic Actuator, Multi-Objective Optimization, Particle Swarm Optimization, scaling law, Preliminary Design

\section{Introduction}

The current technological developments in aviation aim to make aircraft more competitive, greener and safer. The more electric aircraft (MEA) offers promising prospect in terms of performance, maintenance, integration, reconfiguration, ease

\footnotetext{
*Corresponding author; e-mail: wushuai.vip@gmail.com
} 
of operation and management of power (1). Using electricity as the prime source of energy for non-propulsive embedded power systems is one of the most promising way to achieve the above goals.

Electro-hydrostatic actuator (EHA) has been a key power-by-wire (PBW) actuator to replace the traditional hydraulic actuator in MEA $(2,3)$. The block diagram of a typical EHA is shown in Fig. 1. It uses a local servo motor to drive a bi-direction hydraulic pump to control the cylinder. It only requires electric power which can be delivered by cables. MEA using EHA can reduce aircraft weight and maintenance cost by eliminate hydraulic system (4). For example, A380 saves over $450 \mathrm{~kg}$ by introducing two redundancy PBW actuation systesm $(5,6)$

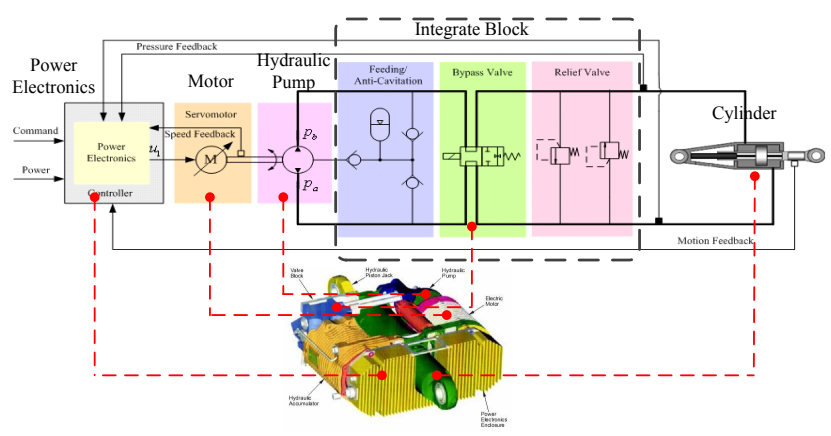

Fig. 1. Electro-hydrostatic actuator (EHA) diagram

Supported by advanced computational tools, early verification and virtual validation of solution in preliminary design can offer significantly cost reduction and quality enhancement over the entire design cycle (7). A simulation-based preliminary design method involving preliminary sizing of electromechanical actuator for an aircraft primary flight control surface(aileron) is proposed in (8). An improved integrated methodology for preliminary design of electromechanical actuators in an European distributed and redundant electromechanical nose gear steering system is given in (9). A modelbased methodology for optimal preliminary design of electro-mechanical actuators is developed to show the usefulness of these estimation models in $(10,11)$. A methodology for the sizing, simulation, analysis, and optimization of electric actuators for the primary and second flight control surfaces of MEA is proposed in $(12,13)$, in which the estimates of control surface flight loads and actuator's weight, framing and solving of the optimization problem and actuator-to-surface allocation are presented.

The design and integration issues of an actuator for MEA should consider several objectives simultaneously. For EHA, the key performance indexes include: light-weight, high efficiency, quick dynamic response and low cost. This means using a multi-objectives optimization (MOO) process to design a EHA will result in more feasible solutions. The presence of MOO in a problem, in principle, gives rise to a set of optimal solutions (mostly known as Pareto-optimal solutions), instead of a single optimal solution (14). The set of Pareto-optimal solutions, usually called Pareto-front is the best performance of all objectives. It helps to provide the preliminary design solution.

In this paper, a MOO methodology for EHA preliminary design is introduced. The aim of this methodology is to search the solutions of three key design parameters of EHA to get the best performances in the preliminary design phase. This study will focus on the two most performances: weight and efficiency. The prediction methods of weight and efficiency based on the main design drivers of the control surface are proposed. A MOO process is carried out to determine the pareto-optimal solutions of weight and efficiency. The results provide compelling evidence of the significant benefits of the proposed method in preliminary design of EHA for MEA, and other similar application. 


\section{Proposed MOO methodology for the preliminary design}

\subsection{Methodology description}

The preliminary design is an important stage in the design cycle of mechatronic system for preliminary size optimization and features synthesis of device parts (10). The inputs are the expective objectives and design constraints from the application requirement. The outputs are design specifications (pump displacement, motor speed, motor torque current coefficient, etc.) and performance index.

The method for the preliminary design of the present study is shown in Fig. 2. Here, the MOO method generates the design parameters based on the input requirements and output to the prediction model. And the prediction models calculate the performance according to the input design parameters. Performance indexes are returned to the MOO method for comparison with the objectives. The intelligence $\mathrm{MOO}$ method will search the parameters to get the optimal solutions by iteration, until the Pareto-front is obtained.

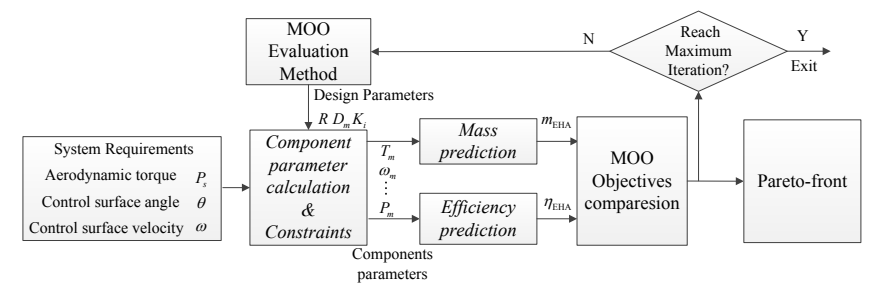

Fig. 2. Proposed MOO methodology process diagram for the preliminary design

\subsection{Choose of design variables and objectives}

The EHA placement and control surface envelope is shown in Fig. 3. The EHA drives control surface rotary by a three rods mechanism. The purpose of preliminary design is to define key parameters of EHA according to the control surface requirements. Requirements of control surface include deflection angle $\left(\delta_{c}\right)$, maximum rotation velocity $\left(\omega_{c}\right)$, and maximum hinge moment $\left(T_{c}\right)$. The first key parameter should be decided is level length $(R)$. Then the required torque of control surface can turn into the specifications of EHA, which include maximum force $(F)$, maximum velocity $(v)$, and linear stroke $(S)$. Using these there parameters, the hydraulic actuator can be designed. Then the displacement of pump should be decided to choose a proper pump. The displacement of pump and the operating pressure can get the torque requirement of motor. The maximum speed requirement of the motor can also be determined by the force and velocity of the hydraulic actuator. The third parameter is about the electric motor of EHA. As the torque and maximum velocity requirement are already determined, then the torque constant $K_{i}$ need be decided. The toque of brushless DC (BLDC) motor equals $K_{i} I$. For a required torque, one can use large $K_{i}$ with small $I$ or small $K_{i}$ with large $I$, and it will influence the weight and efficiency.

In the present study, we aim to find out the optimized $R, D_{m}$ and $K_{i}$ for weight and efficiency, under the control surface requirements.

\section{Prediction and constraint model of EHA}

\subsection{Weight prediction mode}

Weight is one of the most crucial considerations of EHA for MEA applications. Therefore, a weight prediction model is required in preliminary design phase. According to Fig. 1, the weight of EHA includes five parts: the cylinder, pump, 


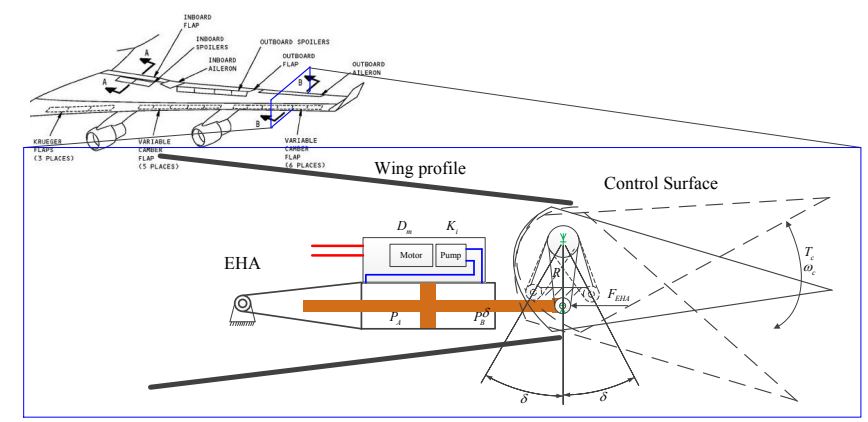

Fig. 3. EHA placement and control surface presentation

motor, integration block and the power electronics. The total weight of EHA equals to

$$
m_{\mathrm{EHA}}=m_{\mathrm{cyl}}+m_{\mathrm{pump}}+m_{\mathrm{motor}}+m_{\mathrm{blk}}+m_{\mathrm{pe}}
$$

where $m_{\text {cyl }}$ is mass of cylinder, $m_{\text {pump }}$ mass of pump, $m_{\text {motor }}$ mass of BLDC, $m_{\text {blk }}$ mass of integration block, and $m_{\text {pe }}$ mass of power block.

The mass of the hydraulic pump and electrical motor is predicted by scaling law. The use of scaling law has the advantage of requiring only one reference component for a complete estimation of a product range $(8,11)$.

Weight prediction of electrical motor The EHA usually uses BLDC motors since the high power-to-mass ratio and good control performance. According to (11), the weight scaling law of BLDC can be express as Eq. (2), which indicates that the motor mass is a power function of torque with index of $3 / 3.5$.

$$
m_{\text {motor }}=a T_{m}^{3 / 3.5}+b
$$

where $a$ and $b$ are two constants which can be found by data fitting. The reference data is the high speed servo motor from Kollmorgen (PMA, PMB, and S series, which rotary speeds are over 4000rpm) (15). The data and prediction equation are shown in Fig. 4.

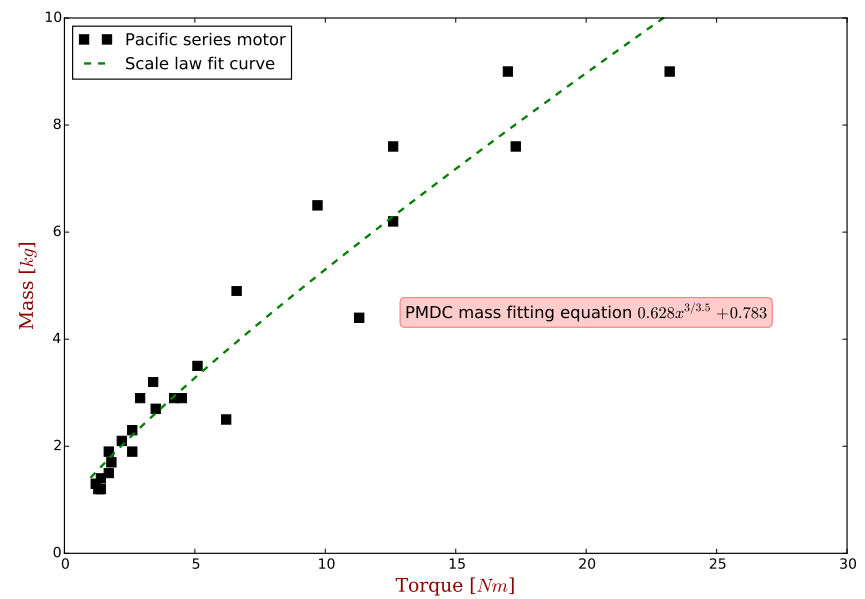

Fig. 4. Mass prediction of BLDC. 
Mass prediction of hydraulic pump The scaling law of axial piston pumps has been studied in (8). In the context of power sizing for preliminary design, the hydraulic pump is considered as a definition parameter. The pump displacement $D_{m}$ represents the geometrical volume of liquid transferred by pump per unit rotation angle. The second parameter influence the mass significantly is the maximum operating pressure $P_{\max }$. High pressure means higher tensile stress from inside and requires thicker wall to make pump reliable. Therefore, the high pressure pump is heavier.

The weight of hydraulic pump is proportional to the displacement of pump which can be presented as

$$
m_{p}=a D_{m}+b
$$

where $D_{m}$ is the displacement of pump, $m_{p}$ is the mass of pump, and $a$ and $b$ are constant coefficients given by fit of collection data. The collection of high speed aerospace hydraulic pumps is from Parker (16), Eaton (17), and MessierBugatti (18). The data and fitting curve, and also the fitting equation and constant coefficients are shown in Fig. 5. The slope of the $35 \mathrm{MPa}$ pump is bigger than the $21 \mathrm{MPa}$ pump as the analysis above.

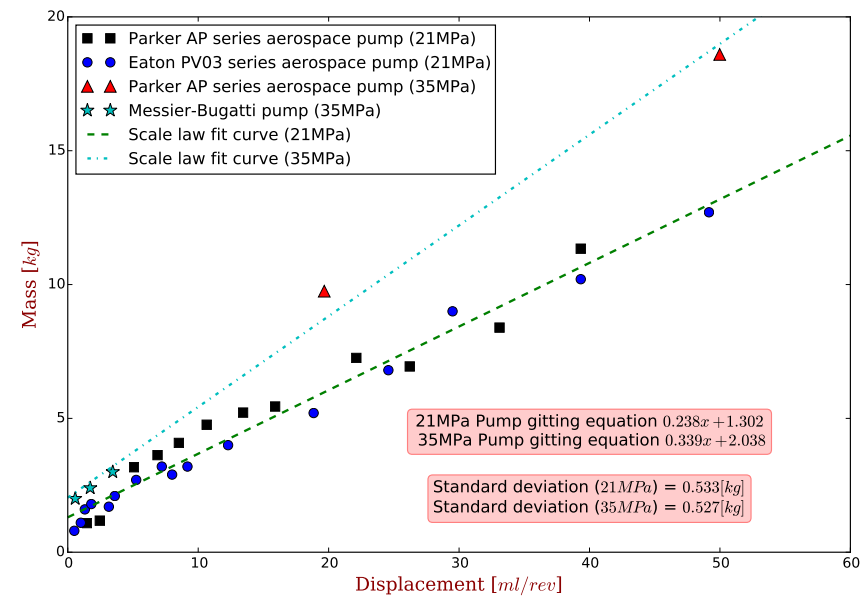

Fig. 5. Pump mass prediction scaling law

Weight prediction of hydraulic cylinder Contrary to the pump and motor, the weight of the hydraulic cylinder is calculated by design parameters and design specification since the cylinder's weight is closely related to the design parameters. The calculation flow chart is shown in Fig. 6. The stall load and the stroke are used to compute the piston-rod diameter $(d)$ based on consideration of buckling, using more conservative of the minimum diameters predicted by Euler and Ranking formula (12). Then the effective piston area $\left(A_{p}\right)$ is computed according to the design of stall load with the operating pressure:

$$
A_{p}=k F_{l} / P
$$

where $k>1$ is a reasonable excess margin. Then the piston outer diameter $(D)$ is computed as $D=\sqrt{d^{2}+4 A_{p} / \pi}$. The required cylinder wall thickness is computed based on circumferential stress with the material's allowable stress. The end-cap thickness is computed using the requirements withstand tensile and shear stresses.

Weight prediction of power electronic The power electrics is to provide appropriate voltage and current to the BLDC. It includes electronics components, such as IGBTs, capacitors, etc. The weight estimation of power electronic presented in (19) is used in here, which is $2.3 \mathrm{~kW} / \mathrm{kg}$. 


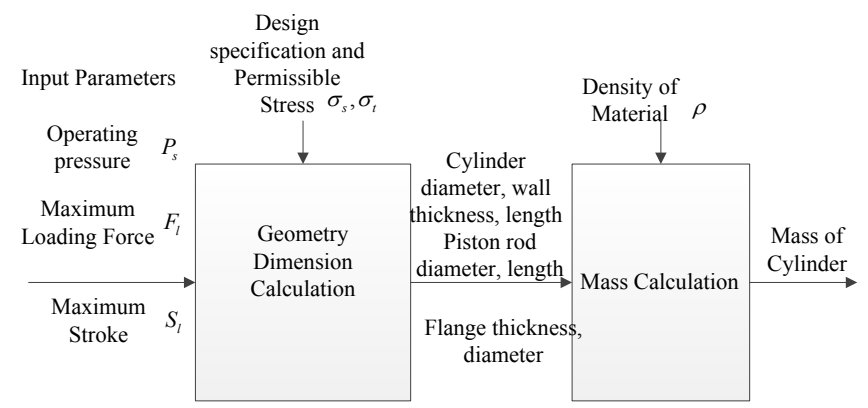

Fig. 6. Cylinder mass calculation flow chart

Weight prediction of integration block The integration block is the frame of EHA and includes the necessary components such as checking valve, filter, and accumulator. The total weight can be estimated by the power of the EHA, which can be presented as

$$
m_{b}=c_{1} P_{\mathrm{EHA}, \max }+c_{2}
$$

where $m_{b}$ is mass of block, $P_{\mathrm{EHA} \text {, max }}$ is maximum power of EHA, $c_{1}$ and $c_{2}$ are two constants.

\subsection{Efficiency prediction}

Efficiency is another important aspect under consideration for EHA design since it not only influences fuel cost but also the thermal characteristics. The efficiency of EHA is an envelope according to output force and output velocity. For simplicity, the efficiency of a typical operating point is selected to represent the efficiency index.

Referring to Fig. 1, the power loss of EHA occurs in power electronics, motor, and pump. Then the efficiency of motor can be presented as

$$
\eta_{\mathrm{EHA}}=\eta_{p e} \eta_{m} \eta_{p}
$$

where $\eta_{p e}$ is the efficiency of power electrics, $\eta_{m}$ is the efficiency of motor, $\eta_{p}$ illustrates the efficiency of pump.

For the motor, the power loss includes copper loss $W_{c}$, eddy current loss $W_{e}$, mechanical viscous loss $W_{d}$, and mechanical friction loss $W_{h}$. Using an equivalent efficiency formula discussed in (20), the equivalent efficiency of BLDC motor in this study is predicted by:

$$
\begin{aligned}
& \eta_{m}=K_{i} I \omega /\left(K_{i} I \omega+W_{c}+W_{e}+W_{d}+W_{h}\right) \\
& W_{c}=I^{2} R_{m}, \\
& W_{e}=K_{e}(B \delta \omega)^{2}, \\
& W_{d}=K_{b}(\omega)^{2}, \\
& W_{h}=T_{f} \omega,
\end{aligned}
$$

where $K_{i}$ is the torque constant of motor, $R_{m}$ is resistance of motor, $B$ is flux density in the yoke, $\delta$ is thickness of silicon steel sheet, $K_{b}$ is damping coefficient of motor, and $T_{f}$ is friction torque.

Since the resistance $R_{m}$ is proportional to voltage constant $K_{e}$, and $K_{i}=K_{e}$ for simplified BLDC motor model, then Eq. (7) can be represented as:

$$
\frac{1}{\eta_{m}}=1+\alpha_{1} \frac{I}{\omega}+\left(\alpha_{2}+\alpha_{3}\right) \frac{\omega}{I}+\alpha_{4} \frac{\omega}{K_{i} I}
$$

where $\alpha_{1}, \alpha_{2}, \alpha_{3}$ and $\alpha_{4}$ are equivalent constant for simplified.

There are two elements of hydraulic pump efficiency: volumetric efficiency and hydro-mechanical efficiency. Assume the viscosity of oil is constant. The volumetric power loss is proportional to operation pressure and inverse proportional to 
pump rotation speed. Hydro-mechanical power loss is proportional to pump speed and dimension of pump as

$$
\eta_{p}=P_{o} D_{m} \omega /\left(P_{o} D_{m} \omega+K_{l e} P_{o}^{2}+K_{b p} \omega^{2}\right)
$$

where $K_{l e}$ is leakage coefficient, $K_{b p}$ is viscous damping coefficient. $K_{l e}$ and $K_{b p}$ are both vary with $D_{m}$. In present study, they are assumed as $K_{b p} \propto D_{m}^{1 / 3}, K_{l e} \propto D_{m}^{(1 / 3)}$. Then the pump efficiency can be simplified as:

$$
\frac{1}{\eta_{p}}=\left(1+\beta_{1} \frac{D_{m}^{(1 / 3)} P_{o}}{D_{m} \omega}+\beta_{2} \frac{D_{m}^{(1 / 3)} \omega}{P_{o} D_{m}}\right),
$$

where $\beta_{1}$ and $\beta_{2}$ are two constant coefficients.

The power loss of power electronics is due to the resistance of electric components. Assume the resistance is constant, then the efficiency of power electrics can be represented as:

$$
\eta_{p e}=1-\gamma_{1} I
$$

Then the efficiency of EHA can be predicted by Eq. (8), (10), and (11) by the design parameters.

\subsection{Constraints}

Constraint of motor The constrains of motor include maximum current and maximum velocity. Maximum current is limited by motor and the electric source both. Since the motor can bear several times stall current for a short period, the maximum current of motor is not considered in present study, and only consider the maximum current limited by electric source.

Motor has mechanical limited and electrical limited maximum velocity. Since the mechanical limited speed of motor for aerospace application is very high, then only the electrical limited is considered in presented study which can be represented by

$$
\omega_{m, \max }=\frac{U}{K_{i}}
$$

Constraint of pump The pump has its operational areas which are limited by operational constraints (11). The most important constraints for pump is the mechanical limit of maximum rotational speed. The scaling law of maximum rotation speed of pump has been given in (8). The maximum speed is power function of displacement with index of $-1 / 3$. Then the fitting equation in present study is defined as

$$
\omega_{p, \max }=a D_{m}^{-1 / 3}+b
$$

where $\omega_{p, \max }$ is maximum rotary speed of pump, $a$ and $b$ are two constants. The data and fitting curve of collection pump data is shown in Fig. 7. The data fits in well with this scaling law. In present study, this scaling law is used as one of the constraints to check whether the solution is realizable.

The constrain is multiplied by a margin factor on the scaling law. It is the envelope curve in the Fig. 7 which equals to multiple of a margin factor to the fitted curve. 


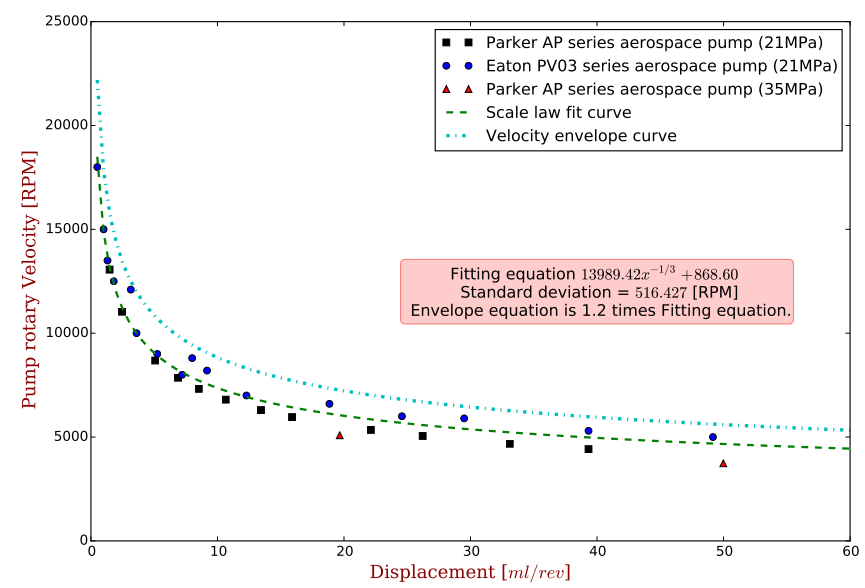

Fig. 7. Pump maximum velocity prediction scaling law

\section{Multi-objective optimization method}

\subsection{MOO problem mathematic description}

The MOO problem can be defined as: let $S \in R^{n}$ be an $n$-dimensional search space, and $f_{i}(x), i=1, \cdots, k$, be $k$ objective functions defined over $S$. Let $\mathbf{f}$ be a vector function defined as

$$
\mathbf{f}(x)=\left[f_{1}(x), f_{2}(x), \cdots, f_{k}(x)\right]
$$

and

$$
g_{i}(x) \leq 0, \quad i=1, \cdots, m
$$

be $m$ inequality constraints. Then, we are interested in finding a solution, $x^{*}=\left(x_{1}^{*}, x_{2}^{*}, \cdots, x_{n}^{*}\right)$, that minimizes $\mathbf{f}(x)$. The objective functions $f_{i}(x)$ may be conflicting with each other, thereby,rendering the detection of a single global minimum at the same point in $S$, impossible. For this purpose, optimization of a solution in multi-objectives problems needs to be redefined properly.

A solution $x$, of the multi-objective optimization problem is said to be Pareto optimal, if and only if there is no other solution, $y$, in $S$ such that $\mathbf{f}(y)$ dominates $\mathbf{f}(x)$. In this case, we also say that $x$ is non-dominant with respect to $S$.

The set of all Pareto optimal solutions of a problem is called the Pareto optimal set, and it is usually denoted as $\mathcal{P}^{*}$.

$$
\mathcal{P} \mathcal{F}^{*}=\mathbf{f}(x): x \in \mathcal{P}^{*},
$$

A Pareto front is convex if and only if, for all $u, v \in \mathcal{P} \mathcal{F}^{*}$ and for all $\lambda \in(0,1)$, there exists a $w \in \mathcal{P} \mathcal{F}^{*}$ such that

$$
\lambda\|u\|+(1-\lambda)\|v\| \geq\|w\|
$$

while it is called concave, if and only if

$$
\lambda\|u\|+(1-\lambda)\|v\| \leq\|w\|
$$

\subsection{Multi-Objectives Particle Swarm Optimization (MOPSO) Method}

There are various methods for MOO problem, such as multi-objectives particle swarm optimization (MOPSO) method (21), Pareto-frontier differential evolution (PDE) approach (22), and fast non-dominated sorting genetic algorithm (NSGA-2) 
method $(23,24)$. These approaches use the concept of Pareto dominance to determine the best positions (leaders) that will guide the searching process.

The (MOPSO) method is adopted here since it has good global searching capability and easy to be applied. It is developed from the PSO method. Contrary to the PSO, the MOPSO should find out the set of non-dominant solutions. Then a repository is introduced into MOPSO. In MOPSO, the non-dominant solutions detected by the particles are stored in a repository. Usually, the number of non-dominant solutions is very big or infinity. But the repository has limited size. Then, if it is full, new solutions are inserted based on the retention criterion, that is, giving priority to solutions located in less crowded areas of the objective space. The search space is divided in hypercubes. Each hypercube is assigned a fitness value that is inversely proportional to the number of particles it contains. The pseudo code of MOPSO method can be described as:

1. Initialize the population $S^{\mathrm{POP}}$

(a) FOR $i=0$ TO $N_{p}\left(N_{p}=\right.$ number of particles $)$

(b) Initialize $S_{i}^{\mathrm{POP}}$

2. Initialize the speed of each particle

(a) FOR $i=0$ TO $N_{p}$

(b) $v_{i}=0$

3. Evaluate each of the particles in $S^{\mathrm{POP}}$

4. Store the positions of the particles that represent non-dominant vectors in the repository $S^{\text {REP }}$

5. Generate hydercube of the search space so far, and locate the particles using these supercubes as the coordinate system where the coordinate of each particle are decided by the values of the objective functions.

6. Initialize the memory of each particles (this memory serves as th guide of through the search space. This memory is also stored in the repository):

(a) FOR $i=0$ TO $N_{p}$

(b) $p_{i}^{\mathrm{BEST}}=S_{i}^{\mathrm{POP}}$

7. WHILE maximum number of cycle has not been reached DO

(a) Compute the speed of each particle using Eq. (19).

(b) Compute the new position of the particles adding the speed produce from the previous step

$$
S_{i}^{\mathrm{POP}}(t)=S_{i}^{\mathrm{POP}}(t)+v_{i}(t)
$$

The velocity update for the $i$-th particle becomes

$$
\begin{aligned}
v_{i}(t+1)=w v_{i}(t) & +c_{1} r_{1}\left(p_{i}^{\mathrm{BEST}}(t)-x_{i}(t)\right) \\
& +c_{2} r_{2}\left(R_{h}(t)-x_{i}(t)\right),
\end{aligned}
$$

where $p_{i}^{B E S T}$ is its best position in searching history and $R_{h}$ is the selected leader (usually selected by classical roulette wheel selection method) from the repository, $w$ is the inertia coefficient of velocity, $c_{1}$ and $c_{2}$ are local and social coefficients, respectively, $r_{1}$ and $r_{2}$ are two random numbers in range $[0,1]$. The best position is updated at each iteration, based on the domination relation between the existing best position of the existing best position of the particle and its new position. 
Table 1: Control surface requirement

\begin{tabular}{ll}
\hline Specifications & Value[Unit] \\
\hline Hinge moment & $7200[\mathrm{Nm}]$ \\
Velocity rate & $60^{\circ} / \mathrm{s}$ \\
Maximum deflection angle & $\pm 25^{\circ}$ \\
\hline
\end{tabular}

\section{Case study and discussion}

The present case study in present study is to optimize a EHA for the control surface with the requirements listed in the Table. 1.

Three constrains are employed:

- $\quad$ Maximum allowable pump velocity versus displacement is shown in Fig. 7.

- $\quad$ Maximum voltage is $U_{\max }=270 \mathrm{VDC}$, maximum pump velocity times $K_{i}$ should small than $U_{\max }$.

- Maximum current is 50A.

The maximum operating pressure is set to $35 \mathrm{MPa}$ then the weight prediction use equation $0.339 D_{m}+2.038[\mathrm{~kg}]$. The efficiency prediction coefficient of Eq. (7) to Eq. (11) are listed below.

- $\quad$ Eq. (8): $\alpha_{1}=58, \alpha_{2}=1 e-4, \alpha_{3}=4.4 e-4, \alpha_{4}=1 e-5$

- $\quad$ Eq. (10): $\beta_{1}=6.5 e-14, \beta_{2}=15.2 e-3$

- $\quad$ Eq. (11): $\gamma_{1}=1 e-3$

The optimization bound of parameters are

- Level length: $R \in[50,250] \mathrm{mm}$,

- Pump displacement: $D_{m} \in[0.2,2] \mathrm{ml} / \mathrm{rev}$,

- Torque or voltage constant of motor: $K_{i}=K_{e} \in[0.1,1] \mathrm{Nm} / \mathrm{A}$ or V/rad/s.

The parameter of MOPSO includes: the number of particle population $N_{p}=300$, maximum repository capacity is 100 , and $w=0.9, c_{1}=c_{2}=1.5$, the maximum iteration count is 250 .

In the two objectives of weight and efficiency, the weight is only decided by the design parameters while the efficiency are different for differential operating point. In this study, the optimization for three different operating points are calculated for comparison, which are

- $\quad$ Case 1: 0.67 maximum load , 0.6 maximum velocity,

- Case 2: 0.3 maximum load, 0.9 maximum velocity,

- $\quad$ Case 3: 0.9 maximum load, 0.3 maximum velocity,

The Pareto-front of optimization results and corresponding design parameters of Case 1 are shown in Fig. 8a and Fig. 8d. Results of Case 2 are shown in Fig. 8b and Fig. 8e. Results of Case 3 are shown in Fig. 8c and Fig. 8f. The same color and same marker in the objective curve and design parameters curve is a solution in Pareto-front. These optimization results indicate that the level length is almost optimized to a certain value for each cases (around 134.5mm for operation point 1 , around $132 \mathrm{~mm}$ for operating point 2, around $134 \mathrm{~mm}$ for operating 3 ). These figures also illustrate that the weight and efficiency are traded off by displacement. Small displacement (red color point) get smaller weight (about $17 \mathrm{~kg}$ but lower efficiency. That is because the damping loss increases with speed, especially for the pump. Comparing the optimization results of the lowest weight and highest weight in the Pareto front of Case 1 show that:

- Lowest weight (red circle): cylinder force $53.475[\mathrm{kN}]$, pump displacement $0.6256[\mathrm{ml} / \mathrm{rev}]$, maximum motor and pump speed 20660[rpm] (which very close to the maximum allowed pump speed 20669[rpm]), maximum torque of pump 
$3.485[\mathrm{Nm}]$, torque constant $0.10169[\mathrm{Nm} / \mathrm{A}]$, maximum current 34.27 [A], weight of motor 2.614 [kg], weight of pump $2.52[\mathrm{~kg}]$, total weight $16.64[\mathrm{~kg}]$, total efficiency 0.448 .

- Highest weight (earth yellow diamond): cylinder force $53.556[\mathrm{kN}]$, pump displacement 1.788 [ml/rev], maximum motor and pump speed $7227[\mathrm{rpm}]$ (the maximum allowed pump speed 14871[rpm]), maximum torque of pump 9.962[Nm], torque constant $0.2473[\mathrm{Nm} / \mathrm{A}]$, maximum current 34.27 [A], weight of motor 5.288 [kg], weight of pump $2.914[\mathrm{~kg}$, total weight $19.71[\mathrm{~kg}]$, total efficiency 0.708 .

The cylinder is almost the same because of the similar level in length. The major difference is the pump displacement. Smaller displacement results in smaller pump and torque requirement, and then the motor will be smaller. But high speed introduces more damping loss and lower efficiency. The torque constant increases with the displacement because the maximum current is limited. The torque should match the displacement to make maximum current in the allowed range.

These figures indicate that the other two cases have the same trend but with different results. Case 1 is medium load and medium velocity, the optimized pump displacement rage is $[0.6,1.8][\mathrm{ml} / \mathrm{rev}]$. Case 2 is large load with low velocity, the pump optimized displacement range is $[0.627,4][\mathrm{ml} / \mathrm{rev}]$. Case 3 is small load with high velocity, the optimized pump displacement range is $[0.628,0.944][\mathrm{ml} / \mathrm{rev}]$. These results indicate that the EHA can be optimized for different operating point. Then it can analyze the working condition and determine which zone is the main working mode for the control surface before optimizing the EHA. For example, the rudder usually bear huge load with low speed, whilst aileron carries lighter load at higher speed.

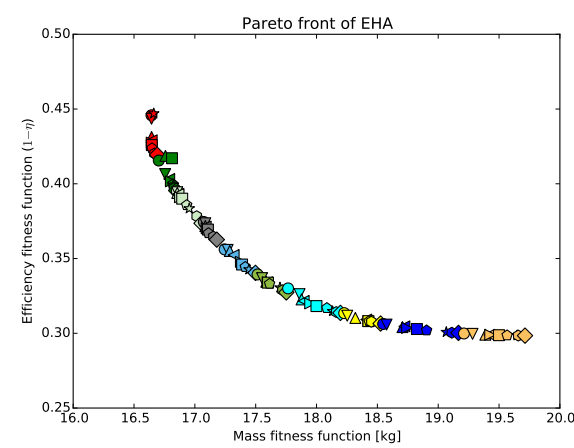

(a) Case 1: Pareto front

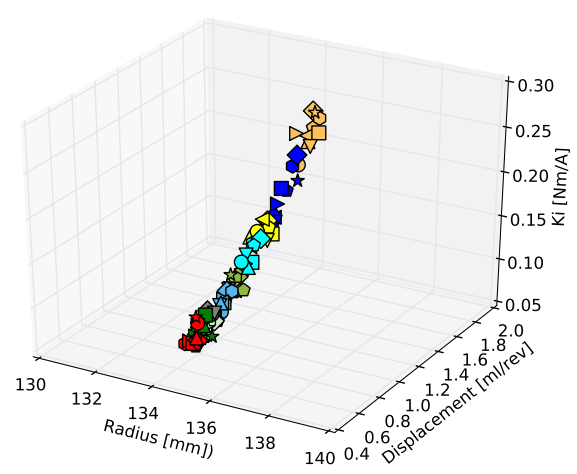

(d) Case 1: Design parameters

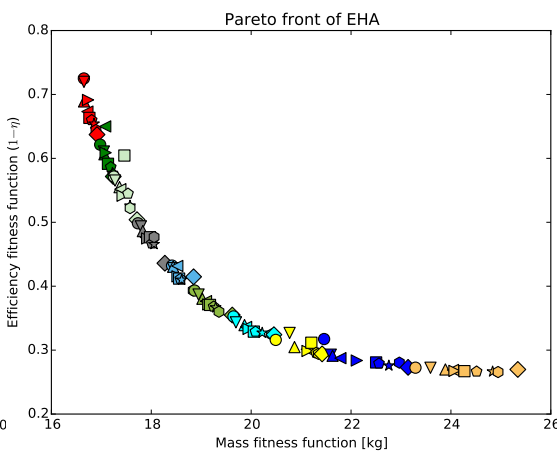

(b) Case 2: Pareto front

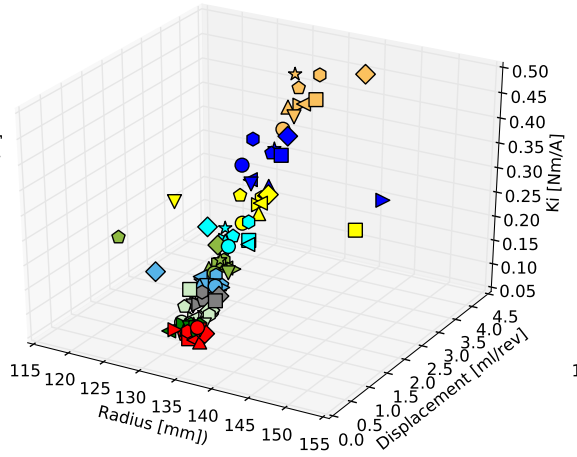

(e) Case 2: Design parameters

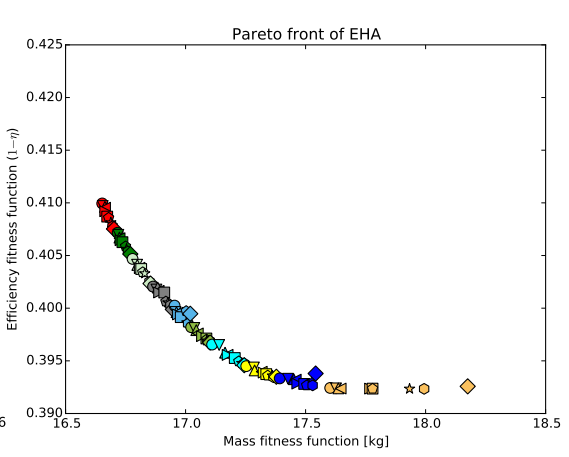

(c) Case 3: Pareto front

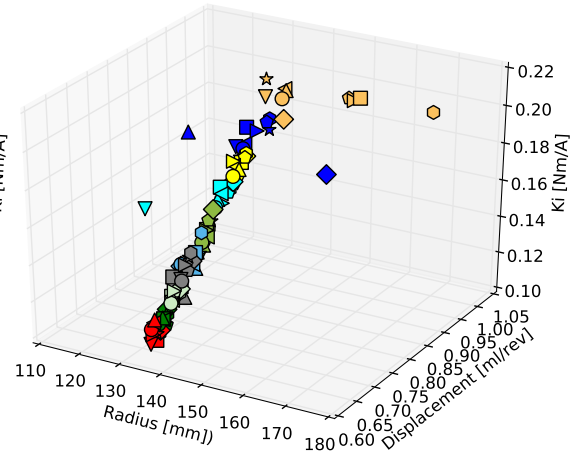

(f) Case 3: Design parameters

Fig. 8. MOO computation results of three different cases.

\section{Conclusion}

A multi-objective optimization preliminary design method has been proposed for preliminary design of EHA. It consider the objective of weight and efficiency, using MOPSO method to get the preliminary design parameters of level length, pump 
displacement, and torque constant of the motor. The weight and efficiency prediction model of EHA has been presented. This methodology has been illustrated with a study case of an EHA with base requirements of control surface. The optimized Pareto-front indicates the performance bound of weight and efficiency. The weight and efficiency are traded-off by the displacement of the pump. The Pareto-front also gives the best design parameters. Further study may involve the prediction of cost, reliability into the objective vector to enhance the preliminary design. It is also envisaged that this method can be applied to other components.

\section{Acknowledgment}

This work is supported by National Key Basic Research and Development Program (2014CB046401) and National Natural Science Foundation of China (No.51235002). This work is supported partially by Chinese School Council, and and Cranfield University who is host to the first author.

\section{References}

[1] Rosero JA, Ortega JA, Aldabas E, Romeral L. Moving towards a more electric aircraft. Aerospace and Electronic Systems Magazine, IEEE. 2007 March;22(3):3-9.

[2] Jensen SC, Dawson D. Flight test experience with an electromechanical actuator on the F-18 Systems Research Aircraft. In: Digital Avionics Systems Conference, 2000. Proceedings. DASC. The 19th. vol. 1; 2000. p. 2E3/1-2E310 vol.1.

[3] Rongjie K, Zongxia J, Shaoping W, Lisha C. Design and simulation of electro-hydrostatic actuator with a built-in power regulator. Chinese Journal of Aeronautics. 2009;22(6):700-706.

[4] Van Den Bossche D. The A380 flight control electrohydrostatic actuators, achievements and lessons learnt. In: Proc. 25th Congress of the International Council of the Aeronautical Sciences; 2006. .

[5] Kulshreshtha A, Charrier J. Electric actuation for flight and engine control: evolution and challenges. In: Presentation on boulder meeting; 2007. .

[6] Chakraborty I, Mavris DN, Emeneth M, Schneegans A. A methodology for vehicle and mission level comparison of More Electric Aircraft subsystem solution: Application to the flight control actuation system. Proceedings of the Institution of Mechanical Engineers, Part G: Journal of Aerospace Engineering. 2014;p. 0954410014544303.

[7] Budinger M, Liscouët J, Maré JC, et al. Estimation models for the preliminary design of electromechanical actuators. Proceedings of the Institution of Mechanical Engineers, Part G: Journal of Aerospace Engineering. 2012;226(3):243-259.

[8] Fraj A, Budinger M, El Halabi T, Maré JC, Negoita GC. Modelling approachs for the simulation-based preliminary design and optimization of electromechanical and hydraulic actuation systems. In: 53rd AIAA/ASME/ASCE/AHS/ASC Structures, Structural Dynamics and Materials Conference 20th AIAA/ASME/AHS Adaptive Structures Conference 14th AIAA. Honoluu, Hawaii; 2012. p. 1523.

[9] Liscouët J, Maré JC, Budinger M. An integrated methodology for the preliminary design of highly reliable electromechanical actuators: Search for architecture solutions. Aerospace Science and Technology. 2012;22(1):9-18.

[10] Budinger M, Reysset A, El Halabi T, Vasiliu C, Maré JC. Optimal preliminary design of electromechanical actuators. Proceedings of the Institution of Mechanical Engineers, Part G: Journal of Aerospace Engineering. 2014;228(9):1598-1616.

[11] Budinger M, Liscout J, Hospital F, Mar JC. Estimation models for the preliminary design of electromechanical actuators. Proceedings of the Institution of Mechanical Engineers, Part G: Journal of Aerospace Engineering. 2012;226(3):243-259. Available from: http: //pig.sagepub.com/content/226/3/243. abstract.

[12] Chakraborty I, Jackson D, Trawick D, Mavris D. Development of a Sizing and Analysis Tool for Electrohydrostatic and Electromechanical Actuators for the More Electric Aircraft. In: 2013 Aviation Technology, Integration, and Operations Conference. Los Angeles, CA: AIAA Aciation; 2013. p. 4282.

[13] Chakraborty I, Trawick D, Jackson D, Mavris D. Electric Control Surface Actuator Design Optimization and Allocation for the More Electric Aircraft. In: 2013 Aviation Technology, Integration, and Operations Conference; 2013. . 
[14] Marler RT, Arora JS. Survey of multi-objective optimization methods for engineering. Structural and multidisciplinary optimization. 2004;26(6):369-395.

[15] High performance Servo Motors; 2006. Available from: http://www.kollmorgen.com/zu-za/products/motors/ discontinued/pmb-series/.

[16] Engine-Driven Pumps;. Available from: http: / /www.parker.com/Literature/.

[17] A Descriptive Summary of Vickers Inline Pumps and their Applications;. Available from: http: / / www . eaton . com/ecm/groups / public/pub/eaton/aero/.

[18] Mini-pumps New generation: a quantum leap in efficiency and competitiveness, all in small, light package;. Available from: www . messier-bugatti.com.

[19] Torabzadeh-Tari M. Dimensioning tools of MEA actuator systems, including modeling, analysis and technology comparison. Kungliga Tekniska Hogskolan; 2008.

[20] Ishikawa T, Tsuji T, Hashimoto S, Kurita N. A simple equivalent circuit for efficiency calculation of brushless DC motors. In: Electrical Machines and Systems (ICEMS), 2013 International Conference on. IEEE; 2013. p. 1133-1138.

[21] Coello CAC, Pulido GT, Lechuga MS. Handling multiple objectives with particle swarm optimization. Evolutionary Computation, IEEE Transactions on. 2004 June;8(3):256-279.

[22] Abbass HA, Sarker R, Newton C. PDE: a Pareto-frontier differential evolution approach for multi-objective optimization problems. In: Evolutionary Computation, 2001. Proceedings of the 2001 Congress on. vol. 2; 2001. p. 971-978.

[23] Deb K, Agrawal S, Pratap A, Meyarivan T. A fast elitist non-dominated sorting genetic algorithm for multi-objective optimization: NSGA-II. Lecture notes in computer science. 2000;1917:849-858.

[24] DEB K. A fast elitist non-dominated sorting genetic algorithm for multi-objective optimization: NSGA-2. IEEE Trans Evol Comput. 2002;6(2):182-197. 